\title{
Gamma Rays from Kaluza-Klein Dark Matter
}

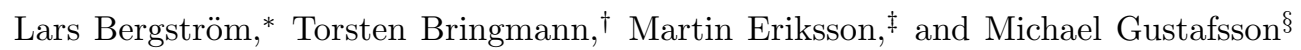 \\ Department of Physics, Stockholm University, AlbaNova University Center, SE - 10691 Stockholm, Sweden
}

(Dated: October 15, 2004)

\begin{abstract}
A TeV gamma-ray signal from the direction of the Galactic center (GC) has been detected by the H.E.S.S. experiment. Here, we investigate whether Kaluza-Klein (KK) dark matter annihilations near the GC can be the explanation. Including the contributions from internal bremsstrahlung as well as subsequent decays of quarks and $\tau$ leptons, we find a very flat gamma-ray spectrum which drops abruptly at the dark matter particle mass. For a KK mass of about $1 \mathrm{TeV}$, this gives a good fit to the H.E.S.S. data below $1 \mathrm{TeV}$. A similar model, with gauge coupling roughly three times as large and a particle mass of about $10 \mathrm{TeV}$, would give both the correct relic density and a photon spectrum that fits the complete range of data.
\end{abstract}

PACS numbers: 95.35.+d, 04.50.+h, 98.70.Rz

\section{INTRODUCTION}

Currently there is great interest in the very high-energy gamma-ray signal from the center of the galaxy, where recently the CANGAROO [1], VERITAS [2] and H.E.S.S. 3] collaborations have reported results. The three experiments find different spectra, something that may depend on different angular acceptances, energy calibrations, etc. The H.E.S.S. result superficially looks more detailed and accurate and is therefore the one we will focus on here, although of course the three groups will have to find a conclusive reason for the reported discrepancies. There have already been speculations of a $\mathrm{TeV}$-scale dark matter (DM) particle giving, through annihilations, a steady source of these photons [3, 4, 5]. Of course, there are other possibilities for emission of these gamma rays related to the physics around the Galactic massive black hole [6].

The prototype DM particle is the neutralino, the lightest supersymmetric particle. The phenomenology of this candidate has been worked out in detail over the last two decades (for a summary, see 7]). However, these models give a rather soft gamma-ray spectrum, so one has to push masses up to the tens of $\mathrm{TeV}$ range to match the results of H.E.S.S., which will be our main concern in this Letter. We focus instead on an interesting alternative which has received much attention in recent years; that of universal extra dimensions (UED) [8, 9, 10]. In these models, all standard model fields propagate in the higher dimensional bulk. For the effective four-dimensional theory, this means that all particles are accompanied by a tower of increasingly more massive Kaluza-Klein (KK) states. Momentum conservation in the extra dimensions, which corresponds to KK mode number conservation, is broken by an orbifold compactification which projects out unwanted degrees of freedom at the zero mode level. If the boundary terms introduced at the orbifold fixed points are identical, then a remnant of KK mode number conservation is left in the form of KK parity, and the lightest KK particle (LKP) is stable [9]. This is anal- ogous to conserved R-parity in supersymmetric models which ensures the stability of the lightest supersymmetric particle. If the LKP is also neutral and non-baryonic, it is a potential dark matter candidate.

We consider the simplest, five-dimensional model with one compactified UED on an $S^{1} / Z_{2}$ orbifold of radius $R$. At tree level, the $n$th KK mode mass is then given by

$$
m^{(n)}=\sqrt{(n / R)^{2}+m_{\mathrm{EW}}^{2}},
$$

where $m_{\mathrm{EW}}$ is the zero mode mass. However, the identification of the LKP is nontrivial because radiative corrections to the mass spectrum of the first KK level are typically larger than the corresponding electroweak mass shifts given by $m_{\mathrm{EW}}$. A one-loop calculation [9] shows that the LKP is well approximated by the first KK mode of the hypercharge gauge boson $B^{(1)}$. The $B^{(1)}$ relic density was determined in 10. Depending on the exact form of the mass spectrum and the resulting coannihilation channels, the limit from the Wilkinson Microwave Anisotropy Probe (WMAP) 11] of $\Omega_{\mathrm{CDM}} h^{2}=0.12 \pm 0.02$ corresponds to $0.5 \mathrm{TeV} \lesssim m_{B^{(1)}} \lesssim 1 \mathrm{TeV}$. Here $\Omega_{\mathrm{CDM}}$ is the ratio of DM to critical density and $h$ is the Hubble constant in units of $100 \mathrm{~km} \mathrm{~s}^{-1} \mathrm{Mpc}^{-1}$. Collider measurements of electroweak observables give a current constraint of $R^{-1} \gtrsim 0.3 \mathrm{TeV}$ [8, 12], whereas LHC should probe compactification radii up to $1.5 \mathrm{TeV}[13]$.

The prospects of KK DM detection have been studied in some detail 14, 15, 16, 17, 18, 19]. Secondary gamma rays from cascading quark decays due to $B^{(1)} B^{(1)}$ annihilation in the GC produce relatively soft spectra [14, 18]. However, unlike the supersymmetric case, charged lepton production is not helicity suppressed, which, e.g., results in a striking peak signal in the positron spectrum [14, 19] for masses $\lesssim 0.5 \mathrm{TeV}$. This has led us to also study the decays of $\tau$ leptons and the spectrum of primary continuum gamma rays from the production and subsequent radiative photon emission of high-energy charged leptons. 


\section{PRIMARY AND SECONDARY GAMMA RAYS FROM $B^{(1)} B^{(1)}$ ANNIHILATIONS}

We first consider primary gamma rays. At tree level, with all other first level KK modes degenerate in mass, $B^{(1)}$ pairs annihilate into quark pairs $(35 \%)$, charged lepton pairs $(59 \%)$, neutrinos $(4 \%)$, charged $(1 \%)$ and neutral $(0.5 \%)$ gauge bosons, and Higgs bosons $(0.5 \%)$. These branching ratios agree with those obtained by Servant and Tait [10] (however, they do not take into account electroweak symmetry breaking). In this Letter we only consider photons radiated from charged leptons $\ell^{ \pm}$. We do not concern ourselves with the radiative processes which electrons and positrons encounter during their propagation through the Galaxy, as these give mostly low-energy photons. The exception may be inverse Compton scattering on infrared photons and starlight, but generally this is expected to be only a small correction [20].

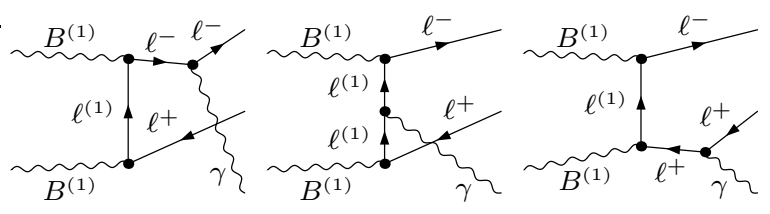

FIG. 1: Contributions to $B^{(1)} B^{(1)} \rightarrow \ell^{+} \ell^{-} \gamma$.

The tree level Feynman diagrams which contribute to the process $B^{(1)} B^{(1)} \rightarrow \ell^{+} \ell^{-} \gamma$ are shown in Fig. [1 The computation of the cross section is straightforward and we find that the differential photon multiplicity is well approximated by

$$
\begin{aligned}
\frac{\mathrm{d} N_{\gamma}^{\ell}}{\mathrm{d} x} & \equiv \frac{\mathrm{d}\left(\sigma_{\ell^{+} \ell^{-} \gamma} v\right) / \mathrm{d} x}{\sigma_{\ell^{+} \ell^{-}} v} \\
& \simeq \frac{\alpha}{\pi} \frac{\left(x^{2}-2 x+2\right)}{x} \ln \left[\frac{m_{B^{(1)}}^{2}}{m_{\ell}^{2}}(1-x)\right],
\end{aligned}
$$

where $x \equiv E_{\gamma} / m_{B^{(1)}}$. From the electromagnetic coupling and the phase space difference between two- and threebody final states, one would expect an answer of the order of $\alpha / \pi$ times a large logarithm, which is related to a collinear divergence. Our calculation shows that there is indeed such a leading logarithmic term, giving large contributions for high photon energies $E_{\gamma}$. Restricting ourselves to these energies (at lower energy, quark fragmentation dominates anyway), the radiative correction, although large, is not more than some five percent of the lowest order cross section. Therefore, there is no need in this first treatment of the problem to sum leading logarithms, but this could eventually be done 21].

We would like to emphasize that our result (21) is almost entirely due to the very large mass of the $B^{(1)}$ and practically independent of the initial state spin. Expressed in the scaling variable $x$, it is furthermore quite

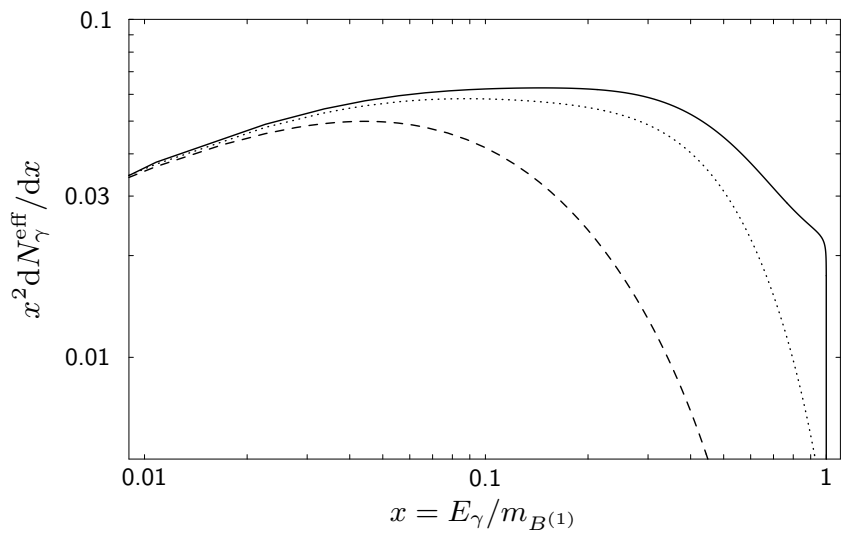

FIG. 2: The total number of photons per $B^{(1)} B^{(1)}$ annihilation (solid line), multiplied by $x^{2}=\left(E_{\gamma} / m_{B^{(1)}}\right)^{2}$. Also shown is what quark fragmentation alone would give (dashed line), and adding to that the contribution from $\tau$ leptons (dotted line). Here we have assumed a $B^{(1)}$ mass of $0.8 \mathrm{TeV}$ and a $5 \%$ mass splitting at the first KK level, but the result is quite insensitive to these parameters.

insensitive to the $B^{(1)}$ mass and the mass splitting at the first KK level. This result therefore applies not only to KK DM, but to any weakly interacting massive particle (WIMP) candidate with nonsuppressed couplings to charged leptons.

Let us now consider secondary gamma rays. As already mentioned, cascading decays of $q \bar{q}$ final states have been studied previously [14, 18]. Here, we also include the semihadronic decays of $\tau$ leptons, which are important in KK DM models since they have a fairly hard spectrum and a branching ratio of around $20 \%$. We will use the recent results of Fornengo, Pieri, and Scopel 22], who have used the Pythia Monte Carlo code 23] to parametrize $\mathrm{d} N_{\gamma}^{q, \tau} / \mathrm{d} x$ for quarks and $\tau$ leptons with a center of mass energy of $1 \mathrm{TeV}$. We neglect the few percent going into $W, Z$, and Higgs final states.

The total number of photons per $B^{(1)} B^{(1)}$ annihilation is given by $\mathrm{d} N_{\gamma}^{\text {eff }} / \mathrm{d} x \equiv \sum_{i} \kappa_{i} \mathrm{~d} N_{\gamma}^{i} / \mathrm{d} x$, where the sum is over all processes that contribute to primary or secondary gamma rays, and $\kappa_{i}$ are the corresponding branching ratios. The result is shown in Fig. 2 Previous analyses of the photon flux correspond to the much softer and sharply falling spectrum from quark fragmentation alone. Also shown is the more important contribution from $\tau$ decays, and of course the radiative direct process which is the main topic of this Letter.

\section{GAMMA-RAY FLUX FROM THE GALACTIC CENTER}

The details of the Galactic halo profile are to a large extent unknown. High resolution N-body simulations favor cuspy halos, with radial density distributions ranging 


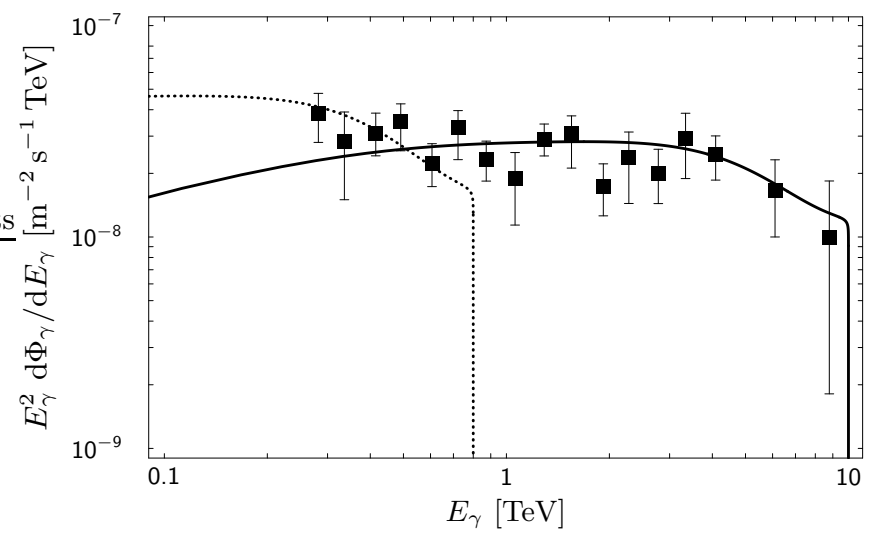

FIG. 3: The H.E.S.S. data 3] compared to the gamma-ray flux from a region of $10^{-5}$ sr encompassing the $\mathrm{GC}$, for a $B^{(1)}$ mass of $0.8 \mathrm{TeV}$, a $5 \%$ mass splitting at the first KK level, and a boost factor $b$ around 200 (dashed line). The solid line corresponds to a hypothetical $10 \mathrm{TeV}$ WIMP with similar couplings, a total annihilation rate given by the WMAP relic density bound, and a boost factor around 1000 .

from $r^{-1}$ [24] to $r^{-1.5}$ [25] in the inner regions. It has further been suggested that adiabatic accretion of DM onto the massive black hole in the center of the Milky Way may produce a dense spike of $r^{-2.4}$ [26]. This has, however, been contested [27, 28]. On the other hand, adiabatic contraction from the dense stellar cluster, which is measured to exist near the GC, is likely to compress the halo DM substantially [29, 30]. This means that there is support for a rather dense halo profile very near the center - something that may be tested with the new 30m-class telescopes 31. Bearing these uncertainties in mind, we will use the moderately cuspy $\left(r^{-1}\right)$ profile by Navarro, Frenk and White (NFW) 24].

Following [32], the differential gamma-ray flux from WIMP annihilation in the GC can be written as

$$
\begin{gathered}
E_{\gamma}^{2} \frac{\mathrm{d} \Phi_{\gamma}(\Delta \Omega)}{\mathrm{d} E_{\gamma}} \simeq 3.5 \cdot 10^{-8} x^{2} \frac{\mathrm{d} N_{\gamma}^{\mathrm{eff}}}{\mathrm{d} x}\left(\frac{\sigma_{\mathrm{tot}} v}{3 \cdot 10^{-26} \mathrm{~cm}^{3} \mathrm{~s}^{-1}}\right) \\
\times\left(\frac{0.8 \mathrm{TeV}}{m_{B^{(1)}}}\right)\left\langle J_{G C}\right\rangle_{\Delta \Omega} \Delta \Omega \mathrm{m}^{-2} \mathrm{~s}^{-1} \mathrm{TeV}, \quad(3)
\end{gathered}
$$

where $\sigma_{\text {tot }} v$ is the total $B^{(1)}$ annihilation rate, and $\left\langle J_{G C}\right\rangle_{\Delta \Omega}$ is a dimensionless line-of-sight integral averaged over $\Delta \Omega$, the angular acceptance of the detector. For a NFW profile, one expects $\left\langle J_{G C}\right\rangle_{\Delta \Omega} \Delta \Omega=0.13 b$ for $\Delta \Omega=10^{-5}$ [33], which is appropriate for the H.E.S.S. telescope. Here, we allow for an explicit boost factor $b$ that parametrizes deviations from a pure NFW profile $(b=1)$ and may be as high as 1000 when taking into account the expected effects of adiabatic compression [30]. For KK DM, $\sigma_{\text {tot }} v \simeq 3 \cdot 10^{-26}\left(0.8 \mathrm{TeV} / m_{B^{(1)}}\right)^{2} \mathrm{~cm}^{3} \mathrm{~s}^{-1}$ [10.

In Fig. 3 we plot the total differential gamma-ray flux for a $B^{(1)}$ mass of $0.8 \mathrm{TeV}$ together with the re- cent H.E.S.S. observations with its surprising, relatively hard spectrum [3]. As can be seen, an excellent fit to the data is obtained for energies up to $m_{B^{(1)}}$, requiring only a moderate value for the boost factor $b$ of roughly 100. A more detailed analysis would of course take the energy resolution of the detector into account, giving a less rapid cutoff at the $B^{(1)}$ mass.

In order to explain the whole observed spectrum with KK DM, one would thus need a mass of $m_{B^{(1)}} \gtrsim 7 \mathrm{TeV}$. Such a high mass, however, is outside the range selected by the relic density constraint and thus disfavored. It has to be kept in mind, though, that models with extra dimensions may have quite a different behavior near freeze-out than ordinary models, which could alter the conclusions about the preferred mass range. Abnormally large running of the $U(1)$ coupling might also allow for larger masses 34].

As remarked before, we expect a similar spectrum for any WIMP dark matter candidate with nonsuppressed couplings to charged leptons. Such a model would give a nice fit to the full H.E.S.S. data, if the canonical WIMP relation given by the WMAP relic density bound, $(\sigma v)_{\text {WIMP }} \sim 3 \cdot 10^{-26} \mathrm{~cm}^{3} \mathrm{~s}^{-1}$ [] , allows for particle masses above $7 \mathrm{TeV}$. For the KK DM scenario considered here, this would require the $B^{(1)}$ 's gauge coupling to be increased by a factor of roughly three. As an illustration, we have included such a hypothetical particle of mass $10 \mathrm{TeV}$ in Fig. [3 with a boost factor of roughly 1000. Models which only get a contribution from quark fragmentation, on the other hand, need a much larger boost factor to fit the observed range. For supersymmetry [5], for example, one is furthermore pushed to a neutralino mass larger than about $12 \mathrm{TeV}$, which starts to involve severe fine-tuning of parameters. It has to be remembered, though, that the radiative effects investigated in this Letter have not yet been systematically studied for supersymmetric models 21].

Of course, the models presented in Fig. [3 must not violate the constraints given by the existing EGRET 35. measurements in the energy range $30 \mathrm{MeV}-10 \mathrm{GeV}$. We find, however, that these upper bounds are easily satisfied. There are some further tests which should be performed in the future to check the KK hypothesis of the TeV gamma-ray signal. Of course, a signal from annihilating DM is not expected to show any detectable time dependence. Since the mass of the DM particle is an abolute cutoff for the energy of any secondary particle, a steplike drop should definitely be searched for. If one gets instruments with better energy resolution, the line from $B^{(1)} B^{(1)} \rightarrow \gamma \gamma$ annihilation [36] may form the final proof of the particle DM hypothesis [32]. It will be interesting to follow the wealth of new results expected from H.E.S.S., as well as MAGIC 37], CANGAROO [1] and VERITAS [2]. The GLAST satellite [38] should also give important data in the range where the various final states cooperate to give a signal. The fate of the 
produced electrons and positrons should also be investigated, something that will need educated guesses for the magnetic field and exact DM density distribution near the GC. In fact, a slightly boosted NFW profile seems to predict a radio signal which is quite close to that observed [18, 20]. We find further that the direct annihilation into neutrinos will, in the KK model and with the same halo parameters explaining the gamma-ray signal, give an event rate corresponding to a few events per $\mathrm{km}^{2}$ per year [18], something that could eventually be detected in a neutrino telescope with good view of the GC.

\section{CONCLUSIONS}

In this Letter we have presented the somewhat surprising result that gamma rays radiated from charged leptons in $B^{(1)} B^{(1)}$ annihilations (e.g., near the GC, but also in other overdense regions of the halo, and maybe even in the diffuse extragalactic gamma-ray spectrum [39]) could be the most promising way to detect the effects of KK DM and to possibly differentiate it from other candidates. We have seen that the signature is a surprisingly hard spectrum, at high energies several orders of magnitude larger than the previously studied case of quark fragmentation alone, which extends all the way to the mass of the DM particle and then drops rapidly. Even if the present gamma-ray signal detected by H.E.S.S. will turn out to have another cause, the sharp drop at $E_{\gamma}=m_{B^{(1)}}$ is a nice signature for that and other experiments to look for.

L.B. is grateful to the Swedish Science Research Council (VR) for support. We acknowledge useful discussions with J. Edsjö and L. Pieri.

* Electronic address: lbe@physto.se

† Electronic address: troms@physto.se

‡ Electronic address: mate@physto.se

$\S$ Electronic address: michael@physto.se

[1] CANGAROO Collaboration, K. Tsuchiya et al., Astrophys. J. 606, L115 (2004), astro-ph/0403592.

[2] VERITAS Collaboration, K. Kosack et al., Astrophys. J. 608, L97 (2004), astro-ph/0403422.

[3] H.E.S.S. Collaboration, F. Aharonian et al., Astron. Astrophys. 425, L13 (2004), astro-ph/0408145.

[4] D. Hooper, I. de la Calle Perez, J. Silk, F. Ferrer and S. Sarkar, JCAP 0409, 002 (2004), astro-ph/0404205.

[5] D. Horns, Phys. Lett. B 607, 225 (2005) [Erratum-ibid. B 611, 297 (2005)], astro-ph/0408192.

[6] F. Aharonian and A. Neronov, Astrophys. J. 619, 306 (2005), astro-ph/0408303.

[7] G. Jungman, M. Kamionkowski and K. Griest, Phys. Rept. 267, 195 (1996), hep-ph/9506380; L. Bergström, Rept. Prog. Phys. 63, 793 (2000), hep-ph/0002126; G. Bertone, D. Hooper and J. Silk, Phys. Rept. 405, 279 (2005), hep-ph/0404175.
[8] T. Appelquist, H. C. Cheng, and B. A. Dobrescu, Phys. Rev. D 64, 035002 (2001), hep-ph/0012100.

[9] H. C. Cheng, K. T. Matchev and M. Schmaltz, Phys. Rev. D 66, 036005 (2002), hep-ph/0204342.

[10] G. Servant and T. M. P. Tait, Nucl. Phys. B 650, 391 (2003), hep-ph/0206071.

[11] D. N. Spergel et al. [WMAP Collaboration], Astrophys. J. Suppl. 148, 175 (2003), astro-ph/0302209.

[12] K. Agashe, N. G. Deshpande and G. H. Wu, Phys. Lett. B 514, 309 (2001), hep-ph/0105084.

[13] H. C. Cheng, K. T. Matchev and M. Schmaltz, Phys. Rev. D 66, 056006 (2002), hep-ph/0205314.

[14] H. C. Cheng, J. L. Feng, and K. T. Matchev, Phys. Rev. Lett. 89, 211301 (2002), hep-ph/0207125.

[15] G. Servant and T. M. P. Tait, New J. Phys. 4, 99 (2002), hep-ph/0209262.

[16] D. Hooper and G. D. Kribs, Phys. Rev. D 67, 055003 (2003), hep-ph/0208261.

[17] D. Majumdar, Phys. Rev. D 67, 095010 (2003), hep$\mathrm{ph} / 0209277$.

[18] G. Bertone, G. Servant, and G. Sigl, Phys. Rev. D 68, 044008 (2003), hep-ph/0211342.

[19] D. Hooper and G. D. Kribs, Phys. Rev. D 70115004 (2004), hep-ph/0406026.

[20] R. Aloisio, P. Blasi and A. V. Olinto, JCAP 0405, 007 (2004), astro-ph/0402588.

[21] L. Bergström, T. Bringmann, M. Eriksson and M. Gustafsson, work in progress.

[22] N. Fornengo, L. Pieri, and S. Scopel, Phys. Rev. D 70 103529 (2004), hep-ph/0407342.

[23] T. Sjöstrand, Comput. Phys. Commun. 82, 74 (1994).

[24] J. F. Navarro, C. S. Frenk and S. D. M. White, Astrophys. J. 462, 563 (1996), astro-ph/9508025.

[25] B. Moore, T. Quinn, F. Governato, J. Stadel and G. Lake, Mon. Not. Roy. Astron. Soc. 310, 1147 (1999), astro-ph/9903164.

[26] P. Gondolo and J. Silk, Phys. Rev. Lett. 83, 1719 (1999), astro-ph/9906391.

[27] P. Ullio, H. S. Zhao and M. Kamionkowski, Phys. Rev. D 64, 043504 (2001), astro-ph/0101481.

[28] M. Milosavljevic and D. Merritt, Astrophys. J. 563, 34 (2001), astro-ph/0103350.

[29] O. Y. Gnedin and J. R. Primack, Phys. Rev. Lett. 93, 061302 (2004), astro-ph/0308385.

[30] F. Prada, A. Klypin, J. Flix, M. Martinez and E. Simonneau, Phys. Rev. Lett. 93, 241301 (2004), astro$\mathrm{ph} / 0401512$.

[31] N. N. Weinberg, M. Milosavljevic and A. M. Ghez, astro$\mathrm{ph} / 0404407$.

[32] L. Bergström, P. Ullio and J. H. Buckley, Astropart. Phys. 9, 137 (1998), astro-ph/9712318.

[33] A. Cesarini, F. Fucito, A. Lionetto, A. Morselli and P. Ullio, Astropart. Phys. 21, 267 (2004), astro-ph/0305075.

[34] K. R. Dienes, E. Dudas and T. Gherghetta, Phys. Lett. B 436, 55 (1998), hep-ph/9803466.

[35] EGRET Collaboration, H. A. Mayer-Hasselwander et al., Astron. Astrophys. 335, 161 (1998).

[36] L. Bergström, T. Bringmann, M. Eriksson and M. Gustafsson, JCAP 0504, 004 (2005), hep-ph/0412001

[37] MAGIC Collaboration, http://wwwmagic.mppmu.mpg.de

[38] GLAST Collaboration, http://www-glast.sonoma.edu/

[39] L. Bergström, J. Edsjö and P. Ullio, Phys. Rev. Lett. 87, 251301 (2001), astro-ph/0105048. 